\title{
Chemical structure peculiarities of different organic matter pools in Haplic Chernozem of the Streleskaya steppe
}

Danchenko N.N. ${ }^{1}$, Artemyeva Z.S. ${ }^{1}$, Kolyagin Yu.G. ${ }^{2}$, Kogut B.M. ${ }^{1}$

${ }^{1}$ Dokuchaev Soil Science Institute, Moscow, Russia, nataly_danch@mail.ru

${ }^{2}$ Lomonosov Moscow State University, Moscow, Russia

Keywords: SOM, granulo-densitometric fractionation, CPMAS ${ }^{13} \mathrm{C}$ NMR, structural features doi: 10.36291/HIT.2019.danchenko.026

Soil organic matter (SOM) is heterogeneous in composition, properties, structure, functions, turnover and mean residence times of its components. Traditionally, the chemical nature of humic substances (humic and fulvic acids) extracted from the soil sample as a whole has been most thoroughly studied. Last decades, there has been increasing interest in the chemical structure of the organic matter of different localization in the soil matrix. In this context, the method of CPMAS ${ }^{13} \mathrm{C}$ NMR spectroscopy is becoming increasingly popular due to the possibility of chemical characterization of low soluble organic substances without destruction.

The aim of our work was to study the chemical composition of OM of different localization in the soil matrix using the CPMAS ${ }^{13} \mathrm{C}$ NMR method. The research subject was the virgin Haplic Chernozem (medium loamy) on the loamy loesses (Streleskaya steppe plot of the Central Chernozemic State Biospheric Reserve, Kursk region).

SOM pools were isolated by a modified variant of granulo-densitometric fractionation, which allows subdividing SOM into four pools: free OM (LFfr), occluded OM (LFocc), OM of clay (Clay) and OM of the residue of soil after the removal of LF and clay (Res).

It is shown that OM pools with different localization in the soil matrix differ significantly in their chemical structure. The data confirm the plant origin of free $\mathrm{OM}$, its chemical structure is the closest to that of plant residues: $\sim 50 \%$ of $\mathrm{C}$ contributes by O-Alkfragments, mainly from carbohydrates with various degrees of polymerization. Aromatic fragments making up $21.8 \%$ of $C$, are presented predominantly by guaiacyl, syringyl and cumaryl structural units of lignin. The decrease in O-alkyl $C$ and the increase in aromatic $C$ in passing from free to occluded $\mathrm{OM}$, indicates a deeper degree of microbiological processing of organic material in the occluded $\mathrm{OM}$, including such resistant to degradation components as cellulose, lignin and tannins. $\mathrm{OM}$ in clay is characterized by higher contribution of O-alkyl C and alkyl C as compared with occluded OM but lower proportion of aromatic fragments (by 2.1 times) and carbonyl groups (by 5.5 times). These structural features indicate the predominantly aliphatic nature of $\mathrm{C}$ associated with clay. Moreover, long-chain structures - the most hydrophobic component of OM predominate among alkyl fragments of clay: the signal intensity at $30 \mathrm{ppm}$ was very high. A sharp increase in the contribution of peptides (1.5 times) along with the alkyl $C$ in clay is apparently due to the presence of microbial products and cellular debris with a high content of alkyl fragments. The spectra of the Res are close to those of Clay, but have noticeable differences: the share of aromatic $C$ in Res increases by 1.4 times, and alkyl, on the contrary, decreases by 1.4 times. Apparently, these differences are due to a greater degree of Res heterogeneity. In addition, the increase in the share of aromatic $C$ may be due to the presence of black carbon components in this fraction.

Revealed structure peculiarities of the different $\mathrm{OM}$ pools, isolated by granulodensitometric fractionation, indicate the specificity of $\mathrm{OM}$ biodegradation processes occurred in various components of soil matrix.

Acknowledgements. The research was provided partly by financial support the Russian Science Academy Presidium (Grant 2018-2020) with the use of the equipment of the Center for Collective Use of V.V. Dokuchaev Soil Science Institute. 